\title{
LETTERS
}

\section{Resident Well-Being and Clinical Teaching Assessments}

\author{
Robert Jackson, MD \\ Louisiana State University Health Science Center, Shreveport, Shreveport, LA, USA.
}

To the Editorsr:- The article by Beckman et al. ${ }^{1}$ highlights the growing importance of learner evaluations of their attending faculty in faculty career advancement. However, a critical problem exists I have yet to see discussed in the literature, namely that of retaliatory evaluations. Our program, as do many, uses a common commercially available computer service to manage evaluations. Learners submit evaluations anonymously, but faculty do not. Also standard is that learners see specific faculty evaluations before submitting their own evaluations of faculty. This has resulted in frequent instances of retaliatory evaluations, often of an extremely vindictive nature. Occasionally faculty have been threatened with sanctions as a result. Another result is that faculty are occasionally afraid to submit less than complimentary evaluations lest the learner becomes angry and submits a retaliatory evaluation, impairing the integrity of evaluations.

Admonishing learners against retaliatory evaluations and punishing those who submit them when they can be identified has not stopped this phenomenon at my institution. One possible response is to not allow learners to see faculty evaluations of themselves before submitting their own. However, this process appears convoluted, and the Office of Medical

Published online May 4, 2010
Education in my department is unwilling to attempt it. This also will not prevent learners who feel they have been unjustly evaluated from submitting a retaliatory evaluation the next time they are assigned to that faculty (which is also common in relatively small programs). Other responses also exist, such as making the entire evaluation process anonymous and using disinterested third parties to provide feedback, but problems also exist with this method, such as the loss of the ability to use specific case examples in the feedback process.

Robert Jackson, MD, Louisiana State University Health Science Center, Shreveport, 1501 Kings Hwy, Shreveport, LA 71130, USA (e-mail: rhjacks@lsuhsc.edu)

\section{REFERENCE}

1. Beckman TJ, Reed DA, Shanfelt TD. Impact of resident well-being and empathy on assessments of faculty physicians. $J$ Gen Intern Med. 2010;25:52-6.

$\mathrm{J}$ Gen Intern Med 25(7):644

DOI: $10.1007 / \mathrm{s} 11606-010-1333-\mathrm{x}$

(C) Society of General Internal Medicine 2010 